\title{
CARACTERIZAÇÃO DE IDOSOS \\ PARTICIPANTES DE PROGRAMAS \\ DE EXERCÍCIOS OFERECIDOS PELO GRUPO \\ DE ESTUDOS E PESQUISAS SOBRE ATIVIDADE FÍSICA PARA IDOSOS - GEPAFI
}

Claudia Beatriz e Silva de Barros Santos ${ }^{1}$

Feng Yu Hua ${ }^{2}$

Marisete Peralta Safons ${ }^{3}$

resumo

O objetivo deste estudo é descrever um grupo de idosos participantes de um programa de atividade física regular, em relação à capacidade funcional, ao perfil sociodemográfico e epidemiológico, utilizando respectivamente: testes funcionais, questões baseadas no "Questionário de Vigilância de Fatores de Risco e Proteção para Doenças

1 Mestra em Educação Física pela Universidade de Brasília (UnB). E-mail: mari7ps@gmail.com.

2 Doutoranda na Universidade de Brasília (UnB). Mestre em Neurociência e Cognição pelo Programa de Pós-Graduação em Neurociência e Cognição da Universidade Federal do ABC. Graduada em Psicologia pela Universidade de Brasília (UnB). Especialista em Neuropsicologia pela Universidade Federal de São Paulo (UNIFESP). E-mail: fengbr2@gmail.com.

3 Professora Doutora Associada lll da Faculdade de Educação Física da Universidade de Brasília (UnB). E-mail: mari7ps@gmail.com. 
Crônicas - VIGITEL" e anamnese de 113 indivíduos, com idade $\geq 60$ anos. Os resultados sociodemográficos verificaram predominância de ( $p=0,001)$ : participação feminina $(74,39 \%)$, casados $(41,46 \%)$, procedência da região sudeste (53,66\%), aposentados (77,50\%), graduados (73,17\%), com plano de saúde particular (76,83\%). Quanto às condições de saúde, todas as faixas etárias apresentaram sobrepeso e pelo menos uma patologia com prescrição de medicação. As patologias mais recorrentes nas mulheres são: problemas visuais, osteopenia a hipertensão arterial; e nos homens: problemas visuais, hipertensão arterial e problema auditivo. Na capacidade funcional, a idade influenciou $(p<0,001)$ no desempenho nos testes de equilíbrio estático e agilidade das mulheres. Nos demais componentes funcionais e na avaliação masculina, não foram encontradas diferenças significativas. Com estes resultados, verificou-se que os idosos que participam de um grupo de atividade física regular, mesmo apresentando pelo menos uma patologia e fazendo uso contínuo de medicamentos, apresentaram uma boa condição física.

palavras-chave

Atividade Física. Capacidade Funcional. Envelhecimento. Saúde.

\section{Introdução}

O crescimento da população idosa está em um nível sem precedentes, acompanhado por transformações demográficas, biológicas, sociais, econômicas e comportamentais (IBGE, 2008; SILVEIRA et al., 2007; VERAS, 2012).

O envelhecimento tem sido foco de diversos estudos científicos, visto a inegável natureza do processo e o impacto do envelhecer em todo mundo. A expectativa de vida aumentou e este acréscimo no número de anos de vida precisa ser acompanhado pela melhoria ou manutenção da saúde e qualidade de vida (WHO, 2005; ACSM, 2009).

Paralelo ao processo de transição demográfica da população ocorre o processo de transição epidemiológica, que se caracteriza pela diminuição da mortalidade, levando ao aumento de doenças crônicas não transmissíveis e comorbidades (PAGOTTO, 2009).

Acredita-se que 85\% dos idosos, mesmo convivendo com algum tipo de problema de saúde, continuam ativos e independentes, e $87 \%$ dos homens idosos brasileiros são chefes de famílias. Destes, mais da metade contribui 
para a renda familiar, mantendo sua importância para o desenvolvimento socioeconômico do país (MINAYO, 2012).

Existe um consenso na literatura sobre a importância da prática de atividades físicas regulares na melhoria das capacidades funcionais do idoso. Os resultados dos estudos sugerem que um estilo de vida ativo pode retardar os impactos do envelhecimento, mantendo assim os idosos ativos independentes para a realização de suas atividades cotidianas por mais tempo em relação a controles não ativos. Da mesma forma, trabalhos utilizando como referência a análise da qualidade de vida, dos fatores de risco cardiovasculares e sociodemográfico, demonstram que o perfil de idosos praticantes de atividade física, apresentam maior porcentagem, apresentando excelentes condições de saúde em relação ao grupo de não praticantes de AF (BORGES; MOREIRA, 2009; MERATI et al., 2011; TORRES et al., 2010).

Pesquisadores que acompanharam o processo do envelhecimento, a partir de grupos de indivíduos, indicam que as práticas corporais realizadas regularmente, proporcionam a manutenção do envelhecimento ativo. Nos grupos, o idoso satisfaz sua necessidade de compartilhar experiências sociais e culturais e amplia seu círculo de contatos sociais, favorecendo a sua inclusão. Estudos apontam para um aumento de programas de atividades físicas, para os idosos, em instituições governamentais e não governamentais. A procura por esses grupos já é uma realidade entre os idosos brasileiros, que atribuem os motivos de sua adesão à busca pela saúde e à socialização (EIRAS et al., 2010; ISER et al., 2011).

Pereira e Okuma (2009) defendem que conhecer o idoso é de fundamental importância para que se possa orientar e atender as suas expectativas. Entretanto, diversas questões relativas ao perfil do idoso praticante de exercícios, permanecem por esclarecer na literatura a fim de que se possa estabelecer o quanto já se conhece do idoso ativo e, a partir disso, poder orientá-los e atender às suas expectativas.

No entanto, existe uma limitação de estudos realizados com idosos residentes no Distrito Federal (DF). Visto que todos os idosos residentes no DF são oriundos de outros estados ou países. Diante deste contexto o presente estudo se justifica pela necessidade de pesquisas relacionadas com idosos residentes no DF. O presente estudo teve como hipótese inicial que os idosos participantes do Grupo de Estudo e Pesquisa sobre Atividade Física para Idosos (GEPAFI) da Universidade de Brasília (UnB) teriam um alto nível socioeconômico e uma boa capacidade funcional.

A realização do presente trabalho justifica-se, portanto, pela necessidade de produzirem-se informações capazes de preencher algumas das lacunas a respeito do idoso inserido no contexto dos grupos de praticantes de atividade física regular. 


\subsection{Delineamento do estudo}

O presente estudo adotou um desenho transversal e descritivo, consistindo na análise das informações do banco de dados obtidos no protocolo de avaliação, que precede o início das atividades semestrais do Programa de Extensão, coordenado pelo GEPAFI da Faculdade de Educação Física da Universidade de Brasília.

Este estudo foi submetido ao Comitê de Ética em Pesquisa (CEP) da Faculdade de Ciências da Saúde (FS) da Universidade de Brasília (UnB) e aprovado sob o número 136/10, conforme a Resolução no 196/96, do Conselho Nacional de Saúde (CNS), que regulamenta as pesquisas envolvendo seres humanos.

Todos os indivíduos, cujos dados atendiam ao critério de inclusão, foram convidados a participar voluntariamente da pesquisa, disponibilizando suas informações para o estudo, mediante assinatura do Termo de Consentimento Livre e Esclarecido, após serem informados e esclarecidos quanto aos objetivos do estudo.

\subsection{Amostra}

O presente estudo adotou um desenho transversal e descritivo, constituído por uma amostra de 113 indivíduos idosos, de ambos os sexos, com idade igual ou superior a 60 anos, sendo 84 mulheres e 29 homens, selecionados através de um levantamento de 2011 do banco de dados com 220 indivíduos matriculados no programa de extensão do GEPAFI.

Foram incluídos neste estudo os indivíduos com idade a partir de 60 anos, regularmente matriculados e frequentes há pelo menos um ano e que tinham realizado todos os testes da bateria de avaliação funcional, respondido ao questionário sociodemográfico e preenchido a anamnese com questões sobre as condições de saúde.

Foram excluídos indivíduos com menos de um ano de matrícula no programa e os que não aceitaram fazer parte do estudo.

\subsection{Instrumentos}

Foram utilizados no estudo: questionário sociodemográfico, anamnese, avaliação antropométrica, e os testes de capacidades funcionais. 
O questionário sociodemográfico foi construído através de questões baseadas no "Questionário de Vigilância de Fatores de Risco e Proteção para Doenças Crônicas - VIGITEL" (MS, 2009) para os dados relativos à identificação do indivíduo, naturalidade, nacionalidade, data de nascimento, idade, endereço, estado civil, sexo, escolaridade, condições de moradia, renda mensal, arranjo familiar, número de filhos, ocupação e domínio de língua estrangeira.

A anamnese consistiu em uma entrevista estruturada, autorreferida e composta de perguntas abertas e fechadas que abordavam: os motivos que levaram o idoso à prática da atividade física como: lazer, convívio social, prescrição médica entre outros, as condições de saúde antes e durante a permanência no programa, modalidade praticada e frequência, ingestão de medicamentos, hábitos relacionados ao estilo de vida atual e passado sobre questões como: alimentação, etilismo e tabagismo.

A antropometria mediu massa corporal, estatura e índice de massa corporal (IMC), utilizando respectivamente: balança marca Plenna, modelo Sport, com resolução de $0,1 \mathrm{~kg}$ e carga máxima de $150 \mathrm{~kg}$; estadiômetro Cardiomed, com resolução de $0,001 \mathrm{~m}$ e estatura máxima de $2 \mathrm{~m}$; razão entre a massa corporal e a estatura ao quadrado $\left(\mathrm{IMC}=\mathrm{kg} / \mathrm{m}^{2}\right)$, sendo classificado em três categorias: normal, sobrepeso e obeso, levando-se em consideração a classificação proposta pela WHO (2005).

Para avaliar a capacidade funcional dos idosos foi realizada uma bateria de testes adaptados para esta faixa etária, composta por seis testes físicos funcionais:

- Flexibilidade (FLEX): teste de sentar e alcançar, no Banco de Wells (ACSM, 2009);

- Equilíbrio (EQ): teste de apoio unipodal com restrição visual, segundo a padronização da bateria de Greene et al. (1993);

- Agilidade (AGI): teste Timed Up and Go (TUG), conforme protocolo de Rikli e Jones (1999);

- Força de Membros Superiores (FMS): dinamômetro Jamar calibrado com escala de 0 a 100kgf, com a padronização citada por Soares e Sessa (1995);

- Força de Membros Inferiores (FMI): teste de sentar e levantar da cadeira em 30 segundos, conforme protocolo de Rikli e Jones (1999);

- Resistência Aeróbia (RA): teste de marcha estacionária foi utilizado para avaliar a resistência aeróbia, conforme protocolo de Rikli e Jones (1999).

As modalidades do programa de treinamento em atividade física do GEPAFI são realizadas duas vezes por semana, com duração de 50 minutos a sessão. A equipe de profissionais é formada por professores e estagiários de Educação Física. 
- Dança de Salão: visa predominantemente o trabalho da potência aeróbia e tem como objetivo atingir a zona alvo de trabalho entre $50 \%$ e $85 \%$ do VO2máx, incrementando a capacidade cardiorrespiratória e coordenação motora, buscando também integração grupal. Os principais exercícios trabalhados na aula foram os de respiração, alongamento e os específicos da dança de salão com deslocamentos e alterações de ritmos (FLETCHER et al., 2001);

- Ioga: trabalha a flexibilidade, o equilíbrio e o controle motor buscando integração das capacidades físicas e mentais. Os principais exercícios trabalhados nas aulas foram os de respiração, alongamento, flexibilidade, resistência muscular, relaxamento e meditação (ALTER, 1999; IYENGAR, 2001);

- Musculação: segue as orientações do ACSM (2009), que consiste em exercícios multiarticulares, priorizando os grandes grupamentos musculares. Trabalhar força nesta faixa etária é indicado prioritariamente para promover incremento na força muscular e na composição corporal, ajudando na redução de peso e aumentando a massa magra. Foram ministrados exercícios resistidos, progressivos e supervisionados.

\subsection{Análise estatística}

Foi utilizada a estatística descritiva para os conjuntos de dados sociodemográficos, condições de saúde e capacidades funcionais.

Na estatística inferencial primeiramente aplicou-se o teste de Shapiro-Wilk para verificar a normalidade na distribuição das variáveis escalares por sexo dentro de cada estrato etário. Em todos os casos obtiveram-se valores indicando que a distribuição não foi normal, optou-se então pelo tratamento não paramétrico com o teste de Kruskal-Wallis para comparação das médias.

Já no tratamento dos dados categóricos utilizou-se o Qui-quadrado $\left(\chi^{2}\right)$ para verificar diferenças entre as frequências encontradas para cada variável.

Em todos os testes foram considerados significantes valores de $p \leq 0,05$ e programa estatístico adotados foi o SPSS 18,0.

\section{Resultados}

Dos 113 idosos participantes do estudo $74,3 \%(n=84)$ eram do sexo feminino e $25,6 \%(n=29)$, do sexo masculino, com idades entre 60 e 84 anos $(69,58 \pm 6,44$ anos). Verificou-se que o número de mulheres foi significativamente $(p=0,001)$ maior que o de homens. 
As tabelas 1 e 2 apresentam os valores descritivos (média \pm desvio padrão) das características antropométricas da amostra estudada. Entre as mulheres, houve ainda diferença estatisticamente significativa $(p=0,02)$ da estatura entre os grupos etários. Verificou-se que as mulheres de 60-64 anos e as acima de 80 anos não diferiram entre si, mas apresentaram estaturas significativamente maiores que as mulheres dos grupos etários de 70-74 anos e de 75-79 anos. Não houve diferenças estatisticamente significativas entre os dados antropométricos dos homens ao se compararem as faixas etárias.

Tabela 1 - Características antropométricas das mulheres por faixa etária.

\begin{tabular}{|c|c|c|c|c|c|c|}
\hline Variáveis & $60-64(27)$ & $65-69(26)$ & $70-74(14)$ & $75-79(11)$ & $\geq 80(6)$ & $p$ \\
\hline $\begin{array}{l}\text { Massa } \\
\text { Corporal (kg) }\end{array}$ & $64,31 \pm 7,82$ & $64,45 \pm 10,56$ & $68,44 \pm 11,29$ & $62,65 \pm 10,36$ & $67,00 \pm 15,51$ & 0,82 \\
\hline Estatura (m) & $157,03 \pm 6,40^{\star}$ & $154,58 \pm 6,29$ & $153,00 \pm 4,52$ & $151,45 \pm 3,59$ & $158,17 \pm 4,54^{*}$ & 0,02 \\
\hline $\mathrm{IMC}\left(\mathrm{kg} / \mathrm{m}^{2}\right)$ & $26,05 \pm 2,63$ & $26,93 \pm 3,8$ & $29,25 \pm 4,69$ & $27,22 \pm 3,69$ & $26,82 \pm 6,41$ & 0,35 \\
\hline
\end{tabular}

Legenda: * = diferença significativa; IMC = índice de massa corporal (IMC $\leq 24,9$ = massa corpórea ideal; $25 \leq \mathrm{IMC}<29,9$ = sobrepeso; $I M C \geq 30$ = obesidade); $\mathrm{p}=$ significância no Teste de Kruskall-Wallis. Fonte: Elaborada pelos autores, 2018.

Tabela 2 - Características antropométricas dos homens por faixa etária.

\begin{tabular}{|c|c|c|c|c|c|c|}
\hline Variáveis & $60-64(3)$ & $65-69(7)$ & $70-74(6)$ & $75-79(8)$ & $\geq 80(5)$ & $p$ \\
\hline $\begin{array}{l}\text { Massa } \\
\text { Corporal (kg) }\end{array}$ & $75,97 \pm 9,34$ & $82,37 \pm 9,85$ & $68,50 \pm 8,43$ & $73,23 \pm 13,44$ & $71,32 \pm 13,93$ & 0,13 \\
\hline Estatura (m) & $168,33 \pm 4,16$ & $170,00 \pm 5,94$ & $162,42 \pm 8,10$ & $168,44 \pm 4,91$ & $165,60 \pm 3,51$ & 0,31 \\
\hline IMC (kg/m²) & $26,74 \pm 2,03$ & $28,49 \pm 2,98$ & $25,94 \pm 2,16$ & $25,73 \pm 3,92$ & $26,09 \pm 5,71$ & 0,32 \\
\hline
\end{tabular}

Legenda: IMC = índice de massa corporal (IMC $\leq 24,9=$ massa corpórea ideal; $25 \leq \mathrm{IMC}<29,9=$ sobrepeso; IMC $\geq 30$ = obesidade); $\mathrm{p}$ = significância no Teste de Kruskall-Wallis.

Fonte: Elaborada pelos autores, 2018.

As características sociodemográficas podem ser observadas na Tabela 3, que compreendem sexo, estado civil, nacionalidade, região de origem, local onde mora, plano de saúde, ocupação social, domínio de língua estrangeira, arranjo familiar, escolaridade e renda, que participam do programa de atividades físicas regular. 
Tabela 3 - Descrição dos aspectos sociodemográficos dos participantes do estudo.

\begin{tabular}{lllc}
\hline Variáveis & $\mathrm{n}$ & $\%$ & $\mathrm{p}$ (Qui-quadrado) \\
\hline Sexo & & & \\
\hline Masculino & 21 & 25,61 & 0,001 \\
Feminino & $61^{\star}$ & 74,39 & \\
\hline
\end{tabular}

\section{Estado Civil}

\begin{tabular}{lccc}
\hline Casado & 34 & 41,46 & \\
Solteiro & $9^{*}$ & 10,98 & 0,001 \\
Viúvo & 23 & 28,05 & \\
Divorciado/Separado & 16 & 19,51 & \\
\hline Região & & & 0,001 \\
\hline Norte & 2 & 2,44 & \\
Nordeste & 22 & 26,83 & \\
Sul & 3 & 3,66 & 0,001 \\
Sudeste & $44^{*}$ & 53,66 & \\
Centro-Oeste & 11 & 13,41 & \\
\hline Plano de Saúde & & & \\
\hline Sim & $63^{*}$ & 76,83 & \\
Não & 19 & 23,17 & \\
\hline Ocupação Social & & & \\
\hline Aposentado & $63^{*}$ & 77,50 & \\
Não Estuda/Não Trabalha & 11 & 13,15 & \\
Trabalha e Estuda & 4 & 4,88 & \\
Trabalha & 4 & & \\
\hline
\end{tabular}

\section{Domínio de Língua Estrangeira}

\begin{tabular}{lccc}
\hline Sim & 23 & 28,06 & 0,001 \\
Não & $59^{*}$ & 71,95 & \\
\hline Escolaridade & & & 0,001 \\
\hline Fundamental & 17 & 20,73 & \\
Médio & 5 & 6,10 & \\
Superior/Pós-Graduação & $60^{*}$ & 73,17 & \\
\hline Renda & & & \\
\hline Até 2 SM & 4 & 4,88 & \\
$>$ 2 a 5 SM & 9 & 10,98 & \\
$>$ 5 a 10 SM & 21 & 25,61 & \\
$>$ 10 a 15 SM & 26 & 31,71 & \\
$>$ 15 a 20 SM & 12 & 14,63 \\
> 20 SM & 10 & 12,19 & \\
\hline
\end{tabular}




\begin{tabular}{lccc}
\hline Variáveis & $\mathrm{n}$ & $\%$ & $\mathrm{p}$ (Qui-quadrado) \\
\hline Arranjo Familiar & & & \\
\hline Cônjuge & 35 & 42,68 & \\
Sozinho & 20 & 24,39 & 0,33 \\
Outros & 27 & 32,93 & \\
\hline
\end{tabular}

Legenda: $n$ = frequência absoluta; $(\%)$ = frequência relativa.

Fonte: Elaborada pelos autores, 2018.

Quanto às características sociodemográficas, a maioria relatou ser casada (41,46\%), de procedência da Região Sudeste (44\%), ter plano de saúde particular (76,83\%), com principal ocupação social a aposentadoria (77,50\%). Quanto à questão da escolaridade, verificou-se que a maioria significativa $(p=0,001)$ dos idosos possuía o diploma de curso superior completo $(73,17)$, o que corresponde a 15 anos de estudos ou mais e a maioria respondeu não dominar outro idioma (59\%).

No que tange à renda familiar a maior porcentagem recebe entre 10 a 15 salários mínimos, seguida pela faixa entre 5 a 10 que foi representada por 23 idosos, e a primeira faixa de intervalo, que é até 2 salários mínimos só atingiu 4 indivíduos.

Ao se analisar o arranjo familiar, a maior parcela dos idosos vive com seus cônjuges ( $42,68 \%)$, seguida pelos idosos morando com os filhos, parentes ou amigos (32,93\%\%) e uma parcela menor morando sozinho $(24,39 \%)$. Não houve diferença significativa $(p=0,33)$ entre os diversos arranjos.

O perfil do grupo em relação às doenças prevalentes está apresentado na Tabela 4. As mais recorrentes nos homens foram: problemas visuais $(86,21 \%)$, a hipertensão arterial (51,72\%) e problema auditivo (34,48\%). Já nas mulheres predominaram: problemas visuais (78,57\%), osteopenia (55,95\%) e hipertensão arterial $(44,05 \%)$.

Não foi verificada diferença estatisticamente significativa nas frequências das diversas doenças prevalentes quando se comparam homens e mulheres em relação a cada doença $(p>0,05)$, exceto para artrose $(p=0,001)$ e osteopenia $(p=0,001)$, que se apresentaram estatisticamente mais frequentes nas mulheres; enquanto os problemas de audição $(p=0,01)$ apresentaram-se estatisticamente mais frequentes nos homens. 
Tabela 4 - Distribuição da amostra segundo tipo de DCNT.

\begin{tabular}{lccccc}
\hline Doenças & MASC & $\%$ & FEM & $\%$ & p (Qui-quadrado) \\
\hline Artrite & 3 & 10,34 & 17 & 20,24 & 0,22 \\
Artrose & 0 & 0 & 33 & 39,29 & 0,001 \\
Diabetes Mellitus & 5 & 17,24 & 11 & 13,1 & 0,58 \\
HAS & 15 & 51,72 & 37 & 44,05 & 0,47 \\
Inc. Urinária & 5 & 17,24 & 14 & 16,76 & 0,94 \\
Labirintite & 4 & 13,79 & 17 & 20,24 & 0,44 \\
Osteopenia & 4 & 13,79 & 47 & 55,95 & 0,001 \\
Osteoporose & 6 & 20,69 & 30 & 35,71 & 0,13 \\
Prob. Audição & 10 & 34,48 & 10 & 11,9 & 0,01 \\
Prob. Coluna & 9 & 31,03 & 35 & 41,67 & 0,31 \\
Prob. Visual & 25 & 86,21 & 66 & 78,57 & 0,37 \\
Stresse & 2 & 6,9 & 13 & 5,48 & 0,24 \\
\hline
\end{tabular}

Legenda: HAS = Hipertensão Arterial Sistêmica; Prob. = problema; Inc. = Incontinência; Masc $=$ masculino; Fem $=$ feminino. Poderia ser registrado mais de um tipo de doença. Fonte: Elaborada pelos autores, 2018.

O perfil do grupo em relação às condições de saúde relacionadas à prevalência de doenças por faixa etária nas mulheres está apresentado na Tabela 5. Verificou-se que nas condições de saúde das mulheres não foram evidenciadas diferenças significativa $(\mathrm{p} \geq 0,05)$ entre as faixas etárias.

Tabela 5 - Características das condições de saúde das mulheres por faixa etária.

\begin{tabular}{lcccccc}
\hline Variáveis & $60-64(27)$ & $65-69(26)$ & $70-74(14)$ & $75-79(11)$ & $\geq 80(6)$ & $\mathrm{p}$ \\
\hline Qtas. Doenças & $3,89 \pm 1,58$ & $4,31 \pm 2,04$ & $5,71 \pm 2,64$ & $4,82 \pm 2,36$ & $6,83 \pm 2,93$ & 0,07 \\
Qto. Medicam. & $1,56 \pm 1,15$ & $2,15 \pm 1,32$ & $2,29 \pm 1,54$ & $2,09 \pm 1,38$ & $3,00 \pm 1,26$ & 0,11 \\
Tempo AF & $3,39 \pm 2,78$ & $5,35 \pm 3,73$ & $4,54 \pm 3,69$ & $3,27 \pm 2,27$ & $7,00 \pm 5,43$ & 0,15 \\
Qto. Pts. Dor & $1,72 \pm 1,29$ & $2,04 \pm 2,03$ & $2,71 \pm 1,94$ & $2,55 \pm 1,13$ & $1,67 \pm 1,63$ & 0,22 \\
\hline
\end{tabular}

Legenda: $\mathrm{p}=$ Kruskal-Wallis; Qtos. = quantos; Pts. = pontos; medicam. = medicamentos.

Fonte: Elaborada pelos autores, 2018.

O perfil do grupo em relação às condições de saúde relacionadas à prevalência de doenças por faixa etária nos homens está apresentado na Tabela 6.

Verificou-se que não foram constatadas diferenças estatísticas $(p \geq 0,05)$ para as variáveis de condições de saúde no grupo masculino. 
Tabela 6 - Características das condições de saúde dos homens por faixa etária.

\begin{tabular}{lcccccc}
\hline Variáveis & $60-64(3)$ & $65-69(7)$ & $70-74(6)$ & $75-79(8)$ & $\geq 80(5)$ & $p$ \\
\hline Qtas. Doenças & $2,67 \pm 2,08$ & $3,29 \pm 2,29$ & $2,67 \pm 1,86$ & $4,25 \pm 2,82$ & $4,00 \pm 1,22$ & 0,46 \\
Qto. Medicam. & $0,67 \pm 0,58$ & $1,57 \pm 1,13$ & $1,33 \pm 1,21$ & $2,13 \pm 1,25$ & $1,20 \pm 1,30$ & 0,53 \\
Tempo AF & $2,67 \pm 2,08$ & $2,86 \pm 1,57$ & $4,00 \pm 1,55$ & $4,88 \pm 2,90$ & $1,80 \pm 0,76$ & 0,13 \\
Qtos. Pts. Dor & $2,00 \pm 1,73$ & $1,29 \pm 1,60$ & $0,67 \pm 0,82$ & $1,13 \pm 1,13$ & $0,40 \pm 0,55$ & 0,65 \\
\hline
\end{tabular}

Legenda: $\mathrm{p}=$ Kruskal-Wallis; Qtos. = quantos; Pts. = pontos; medicam. = medicamentos.

Fonte: Elaborada pelos autores, 2018.

O perfil do grupo feminino em relação às capacidades funcionais por faixa etária está apresentado na Tabela 7.

$\mathrm{Na}$ bateria de testes para capacidade funcional foram encontradas diferenças significativas somente nos testes de equilíbrio estático e dinâmico. Os melhores valores ocorreram na faixa etária de 60 a 64 anos, demonstrando que as mulheres idosas do grupo de menor faixa etária parecem ter melhores resultados para as variáveis acima mencionadas.

Para os demais testes as diversas faixas etárias tiveram resultados estatisticamente semelhantes.

Tabela 7 - Características das capacidades funcionais das mulheres por faixa etária.

\begin{tabular}{|c|c|c|c|c|c|c|}
\hline Variáveis & $60-64(27)$ & $65-69(26)$ & $70-74(14)$ & $75-79(11)$ & $\geq 80(6)$ & $\mathrm{p}$ \\
\hline Flex (cm) & $28,68 \pm 7,33$ & $28,07 \pm 9,31$ & $26,74 \pm 5,85$ & $27,26 \pm 10,22$ & $25,10 \pm 10,71$ & 0,91 \\
\hline $\mathrm{Eq}(\mathrm{s})$ & $9,25 \pm 6,94$ * & $6,66 \pm 5,80$ & $3,47 \pm 2,02$ & $3,27 \pm 2,07$ & $2,95 \pm 1,32$ & 0,00 \\
\hline TUG (s) & $5,43 \pm 0,73$ * & $5,61 \pm 0,78$ & $6,25 \pm 0,88$ & $6,66 \pm 1,07$ & $6,68 \pm 0,92$ & 0,00 \\
\hline FMl (rep.) & $17,87 \pm 4,32$ & $18,69 \pm 4,90$ & $16,29 \pm 5,76$ & $14,00 \pm 4,90$ & $14,40 \pm 3,91$ & 0,06 \\
\hline $\begin{array}{l}\text { FMS dir. } \\
\text { (kgf) }\end{array}$ & $21,93 \pm 5,22$ & $21,42 \pm 4,95$ & $20,43 \pm 5,68$ & $19,27 \pm 4,84$ & $21,00 \pm 2,45$ & 0,67 \\
\hline $\begin{array}{l}\text { RA } \\
\text { (n passos) }\end{array}$ & $\begin{array}{c}118,42 \pm \\
38,63\end{array}$ & $\begin{array}{c}123,73 \pm \\
34,19\end{array}$ & $\begin{array}{c}125,54 \pm \\
49,88\end{array}$ & $\begin{array}{c}109,45 \pm \\
35,21\end{array}$ & $\begin{array}{c}118,20 \pm \\
34,41\end{array}$ & 0,71 \\
\hline
\end{tabular}

Legenda: $p=$ Kruskal-Wallis; Flex = flexibilidade; $\mathrm{cm}$ = centímetro; Eq = Equilíbrio; TUG = agilidade; $\mathrm{FMl}$ = força de membros inferiores; FMS = força de membros superiores; RA = resistência aeróbia; $n^{\circ}=$ número.

Fonte: Elaborada pelos autores, 2018.

O perfil do grupo masculino em relação às capacidades funcionais por faixa etária está apresentado na Tabela 8.

Na bateria de testes para capacidade funcional não foram encontradas diferenças significativas entre as faixas etárias. 
Tabela 8 - Características das capacidades funcionais dos homens por faixa etária.

\begin{tabular}{lcccccc}
\hline Variáveis & $60-64(3) \#$ & $65-69(7)$ & $70-74(6)$ & $75-79(8)$ & $\geq 80(5)$ & $p$ \\
\hline Flex (cm) & $20,23 \pm 12,45$ & $17,01 \pm 12,10$ & $28,63 \pm 33,62$ & $18,63 \pm 6,64$ & $22,22 \pm 18,25$ & 0,94 \\
Eq (s) & $10,74 \pm 13,09$ & $5,16 \pm 4,35$ & $10,70 \pm 11,55$ & $3,95 \pm 2,51$ & $15,04 \pm 24,19$ & 0,46 \\
TUG (s) & $5,01 \pm 0,50$ & $5,53 \pm 1,82$ & $5,66 \pm 0,57$ & $5,66 \pm 0,68$ & $6,03 \pm 1,19$ & 0,23 \\
FMI (rep.) & $17,33 \pm 1,53$ & $16,21 \pm 4,40$ & $22,17 \pm 3,92$ & $22,00 \pm 5,73$ & $15,60 \pm 4,34$ & 0,07 \\
FMS (kgf) & $37,33 \pm 5,03$ & $44,29 \pm 20,20$ & $40,83 \pm 19,68$ & $31,50 \pm 6,99$ & $32,20 \pm 7,43$ & 0,14 \\
& $113,00 \pm$ & $112,50 \pm$ & $127,17 \pm 9,28$ & $128,88 \pm$ & $114,40 \pm$ & 0,30 \\
RA (nopassos) & 19,16 & 12,93 & & 19,98 & 23,20 & \\
\hline
\end{tabular}

Legenda: $p=$ Kruskal-Wallis; Flex = flexibilidade; $\mathrm{cm}=$ centímetro; $\mathrm{Eq}=$ Equilíbrio; $T \mathrm{TG}$ = agilidade; $\mathrm{FMl}=$ força de membros inferiores; FMS = força de membros superiores; RA = resistência aeróbia; n = número; \# = excluído do tratamento inferencial.

Fonte: Elaborada pelos autores, 2018.

\section{Discussão}

\subsection{Perfil sociodemográfico}

Os resultados deste estudo indicam que a média da idade do grupo estudado (69,58 anos) está próxima à expectativa máxima de vida do brasileiro na atualidade que é de 73,1 anos de vida em média (IBGE, 2010).

Em relação ao IMC, todos os grupos etários masculinos e femininos foram classificados na categoria de sobrepeso: de 25 a $30 \mathrm{~kg} / \mathrm{m} 2$ (Halpern, 1999). Porém, tem que se considerar possíveis diferenças na classificação do IMC para idosos, uma vez que os valores não são específicos para essa faixa etária, pois o envelhecimento é caracterizado por modificações biológicas particulares (BENEDETTI; MEURER; MORINI, 2012).

Quanto à proporção de gênero da população estudada os resultados encontrados foram de $25,6 \%$ de homens e $74,3 \%$ de mulheres, o que é esperado, em uma população idosa, devido a maior expectativa de vida entre as mulheres (KUHNEN, 2008; IBGE, 2010). Corroborando vários achados da literatura (PEREIRA; OKUMA, 2009; ANTUNES; MAZO; BALBÉ, 2011; VIRTUOSO et al., 2012), que verificam a predominância da participação feminina em relação a masculina em programa de atividade física. Esta evidência pode estar relacionada a mortalidade diferenciada de gêneros que prevalece há muitos anos entre os brasileiros (SOBREIRA; SARMENTO; OLIVEIRA, 2011). 
Sobre o estado civil, a maioria dos idosos pesquisados relatou estar casado (41,5\%), de forma semelhante aos achados de Mota et al. (2006), Cipriani et al. (2010), Antunes, Mazo e Balbé (2011) e Virtuoso et al. (2012), que encontraram respectivamente $65,2 \%, 39,1 \%, 55,2 \%$ e $55,1 \%$. Este achado difere dos dados que indicam maior índice para a viuvez no Brasil e nas Américas (VERAS, 2003; SOBREIRA; SARMENTO; OLIVEIRA, 2011).

Quanto ao grau de instrução, os resultados apresentaram um nível elevado de escolaridade (73,1\% com nível superior ou pós-graduação completo) em relação ao Censo Demográfico de 2010 que foi de 3,3 anos de estudo para a média da população de idosos no Brasil. O alto nível de escolaridade verificado neste estudo é uma característica marcante no perfil dos participantes do GEPAFI. Pesquisas realizadas em países desenvolvidos mostram que a maior adesão aos programas de exercício físico está associada com níveis de escolaridade e de renda mais alta (HOMBERGH et al., 1995; FLORINDO et al., 2001).

Os idosos do Distrito Federal são procedentes de outras regiões do Brasil, em decorrência das correntes migratórias geradas pela oferta de trabalho criada na transferência da capital federal para Brasília. A região Sudeste é a mais representativa, onde 23,5\% provem do estado de Minas Gerais e 18,8\% estado do Rio de Janeiro. Em 2007, Danilow et al. verificaram que a maior parte de idosos institucionalizados do Distrito Federal eram procedentes da região Nordeste, estes dois achados que podem parecer contraditórios, na verdade podem estar sofrendo a influências socioeconômicas que inteferem na autonomia.

A maioria dos idosos apresentou renda mensal acima da população brasileira, de 10 a 15 salários mínimos (31,71\%), seguida de idosos que recebiam entre 5 a 10 salários mínimos (25,61\%). A média da renda familiar apresentada pelo IBGE (2010) que é até 3 salários mínimos. Virtuoso et al. (2012), também verificaram uma média de 5 salários mínimos (46,7\%), quando foi estudado o perfil de morbidade referida e padrão de acesso a serviços de saúde por idosos praticantes de atividade física.

Quanto ao contexto familiar, $40 \%$ dos idosos desse estudo moram com seus cônjuges, $26,8 \%$ deles moram sozinhos e $23 \%$ moram em companhia dos filhos. Corroborando com Mota et al. (2006), que em seu estudo sobre qualidade de vida associada à saúde em idosos participantes e não participantes em programas regulares de atividade física, relataram que no grupo ativos a maioria era mulheres idosas jovens $(65,2 \%)$, casadas $(56,5 \%)$, viviam com seus companheiros ou outro $(71,7 \%)$ e que $28,3 \%$ moravam sozinhos.

Com relação à utilização e cobertura de plano de saúde, a maioria dos idosos (81,7\%) relatou ter cobertura por plano de saúde, para $14,6 \%$ declararem não ter. Segundo Virtuoso et al. (2012), relataram que com o aumento da idade, os idosos utilizaram mais a rede privada de assistência à saúde. 


\subsection{Condições de saúde}

Todos os idosos que fizeram parte do estudo apresentaram doenças crônicas, sendo mais prevalentes os problemas visuais, presentes em $78,57 \%$ das mulheres e $86,21 \%$ dos homens e a hipertensão arterial, relatada por $44,05 \%$ das mulheres e $51,72 \%$ dos homens.

Em estudo apresentado por Virtuoso et al. (2012) sobre o perfil de morbidades referidas e padrão de acesso a serviços de saúde por idosos praticantes de $\mathrm{AF}(\mathrm{n}=165 ; 132 \mathrm{M}$ e $33 \mathrm{H}), 69,6 \%$ relataram possuir cobertura por plano de saúde e $87,2 \%$ dos idosos pesquisados referiram pelo menos uma doença crônica não transmissíveis diagnosticada por profissional qualificado. As morbidades relatadas de maiores prevalências foram: a hipertensão $(48,4 \%)$, as doenças osteoarticulares (47,7\%), e a hipercolesterolemia (32,7\%).

Da mesma maneira, Krug et al. (2012) analisando as condições de saúde de 27 idosos longevos, sendo 19 mulheres e 8 homens, que participaram de um programa de atividade física, identificaram que 92,6\% desta amostra apresentaram alguma doença crônica não transmissível e que 88,9\% faziam uso continuamente de medicamentos. Verificaram também que a doença de maior prevalência foi a hipertensão (56\%), seguida pela hipercolesterolemia (28\%) e pelas doenças cardíacas $(28 \%)$.

Na comparação entre homens e mulheres foram verificadas frequências superiores estatisticamente significativas nos diagnósticos de artrose $(p=0,001)$ e osteopenia $(\mathrm{p}=0,001)$ para as idosas, concordando com dados normativos que afirmam serem as doenças osteoarticulares de 3 a 5 vezes mais frequentes população feminina (Nieman, 1999). Na masculina, os problemas de audição $(p=0,01)$ apresentaram maior frequência.

\subsection{Capacidade funcional}

Analisando os componentes da capacidade funcional e considerando as diferentes faixas etárias, não foi verificada diferença significativa em flexibilidade, força de membros inferiores e resistência aeróbia das mulheres, sugerindo a manutenção dessas habilidades mesmo no envelhecimento com a prática de atividade física como estratégia para a preservação das funções físicas, adiando e podendo reverter os efeitos deletérios desta fase do desenvolvimento humano (ZAGO, 2010).

No presente estudo com exceção da força de membros inferiores, os homens da faixa etária de 60 a 69 anos apresentaram desempenho inferior que o das mulheres da mesma idade na faixa etária dos 60 aos 69 anos em todas 
as variáveis. Já na faixa de 60 a 64 anos, os homens mostraram-se melhor na força de membros superiores e equilíbrio mais fortes que as mulheres.

Mazo et al. (2010) ao avaliarem 112 idosos, verificaram que quando comparados às idosas com características semelhantes, os homens obtiveram resultados inferiores nos testes de força, agilidade/equilíbrio dinâmico e coordenação. Já no teste de capacidade aeróbia os resultados encontrados foram maiores para os homens idosos, mas quando as médias de homens e mulheres foram comparadas os resultados não diferenciaram estatisticamente.

Streit et al. (2011) avaliaram a aptidão física de homens e mulheres separadamente utilizando os mesmos testes à do presente trabalho para as variáveis: flexibilidade, agilidade e força de membros inferiores, agilidade e for, com a diferença no relato dos resultados que foi apresentado em cinco categorias: muito ruim, ruim, regular, bom e muito bom. Ao se compararem os resultados por sexo e por faixa etária, verificou-se que a amostra do presente trabalho está classificada na faixa de rendimento que os autores do estudo classificaram como muito bom para flexibilidade, regular para agilidade e de regular para muito bom para força de membros inferiores.

Outro padrão verificado por Nunes et al. (2009) e por Borges e Moreira (2009) foi o do declínio das diversas capacidades funcionais com o avançar das faixas etárias, observação que está de acordo com o padrão já estabelecido na literatura. No presente estudo este padrão não foi verificado para os homens em nenhuma variável e para as mulheres o declínio só ocorreu para a variável equilíbrio, que decresceu significativamente conforme os grupos de estratos superiores da faixa etária foram comparados com os inferiores. Nunes e Santos (2009) encontraram resultados semelhantes ao compararem o desempenho de 113 idosos praticantes de caminhada, hidroginástica e lian gong.

O presente estudo avaliou também faixas etárias de estratos acima dos 70 anos e mensurou um número maior de variáveis do condicionamento físico, o padrão de superioridade por gênero não foi consistente. Apenas dois padrões foram mantidos para todas as faixas: em agilidade e equilíbrio dinâmico as mulheres obtiveram maiores valores em todas as faixas etárias, já em força de membros superiores, os homens é que apresentaram maiores valores em todas as faixas etárias. Nas demais variáveis o que se verificou foi a alternância entre os gêneros.

Em relação à amostra feminina, os resultados correspondentes à flexibilidade, agilidade e força de membros inferiores se mostraram compatíveis com os parâmetros sugeridos por Rikli e Jones (2012), para a manutenção da independência funcional de idosos, fundamental para a qualidade de vida e da saúde no envelhecimento. 
Os dados deste estudo corroboram a correlação positiva entre autonomia e capacidade funcional, pois os resultados obtidos na amostra deste estudo apresentam médias iguais ou superiores às sugeridas pelas por Rikli e Jones (2012), considerando que todos os participantes da pesquisa assumem as atividades cotidianas com autonomia e independência.

\section{Conclusão}

O perfil sociodemográfico dos idosos participantes de um programa de atividade física regular deste estudo revelou que o idoso ativo do Distrito Federal é predominantemente do sexo feminino, com a maioria na faixa etária de 65 a 69 anos, e com uma renda mensal entre 10 a 15 salários mínimos.

Em relação ao perfil das condições de saúde o idoso ativo do Distrito Federal apresentou-se tanto para os homens como para as mulheres com um percentual grande de problemas visuais e hipertensão arterial. E nas mulheres um número grande com osteopenia e nos homens problemas de audição.

$\mathrm{O}$ idoso do Distrito Federal demonstrou capacidades funcionais iguais ou superiores aos encontrados na literatura, definindo condições adequadas para manutenção da autonomia, configurando um fator protetor para algumas perdas inerentes ao envelhecimento.

Com os resultados apresentados verificou-se o perfil epidemiológico, sociodemográfico e o nível da capacidade funcional dos idosos que participam de um grupo de atividade regular na Universidade de Brasília. Embora os participantes tenham relatado pelo menos uma patologia com uso contínuo de medicamentos, suas condições físicas se mostraram satisfatórias.

De modo geral, os resultados deste estudo estão condizentes com os dados encontrados na literatura nacional e este trabalho soma evidências que fortalecem a tendência de se encontrarem indivíduos cada vez mais vigorosos, com boa saúde e integrados socialmente entre os idosos praticantes de atividades físicas. Entretanto, ainda existem lacunas em relações a possíveis correlações entre fatores sociodemográficos, condições de saúde e capacidades funcionais destes idosos ativos que necessitam de estudos adicionais para serem elucidados. 
CHARACTERIZATION OF ELDERLY

PARTICIPANTS OF EXERCISE PROGRAMS

OFFERED BY THE GROUP OF STUDIES AND

RESEARCH ON PHYSICAL ACTIVITY FOR

ELDERLY - GEPAFI

\section{abstract}

The objective of this study is to describe a group of elderly participants in a program of regular physical activity in relation to functional capacity, the sociodemographic and epidemiological profile, using respectively: functional testing, issues-based "Survey of Risk Factors Surveillance and Protection Chronic Disease - VIGITEL "and anamnesis of 113 individuals aged $\geq 60$ years. The results showed demographic predominance ( $p=0.001)$ : participation of women (74.39\%), married (41.46\%), coming from the southeast region (53,66\%), retired (77.50\%), college (73.17\%) and coverage of private health insurance (76.83\%). Regarding health, all age groups were overweight and at least one condition with some medication prescription. The most frequent pathologies in women are: visual problems, osteopenia and hypertension; and in men: visual problems, hypertension and hearing problem. Regarding functional capacity, age had a significant influence $(p<0.001)$ in performance on tests of static balance and agility of women. In the other functional components and regarding male evaluation, no significant differences were found. With these results, it was found that older adults who participate in a regular group of physical activity, even presenting at least one condition and making use of continuous medication, had a good physical condition.

keywords

Physical Activity. Functional Capacity. Age. Health.

referências

ALTER, Michael. Ciência da Flexibilidade. 2. ed. Porto Alegre: Artmed, 1999.

AMERICAN COLLEGE OF SPORTS MEDICINE (ACSM). Exercise and physical activity for older adults. Medicine and Science in Sports and Exercise, Indianapolis, v. 41, n. 7, p. 1510-1530, 2009.

ANTUNES, Giselle; MAZO, Giovana; BALBÉ, Giovane. Relação da autoestima entre a percepção de saúde aspectos sociodemográficos de idosos praticantes de exercício físico. Revista da Educação Física, Maringá, v. 22, n. 4., p. 583-589, out./dez. 2011. 
BENEDETTI, Tânia; MEURER, Simone; MORINI, Simone. Índices antropométricos relacionados a doenças cardiovasculares e metabólicas em idosos. Revista da Educação Física, Maringá, v. 23, n. 1, p. 123-130, 2012.

BORGES, Milene; MOREIRA, Ângela. Influências da prática de atividade físicas na terceira idade: estudo comparativo dos níveis de autonomia para o desempenho nas AVDS e AIVDS entre idosos ativos fisicamente e idosos sedentários. Motriz, Rio Claro, v. 15, n. 3, p. 562-573, jul./set. 2009.

CIPRIANI, Natália et al. Aptidão funcional de idosas praticantes de atividades físicas. Revista Brasileira de Cineantropometria e Desempenho Humano, Florianópolis, v. 12, n. 2, p. 106-111, 2010

DANILOW, Milena et al. Perfil epidemiológico, sociodemográfico e psicossocial de idosos institucionalizados do Distrito Federal. Comunicação em Ciências da Saúde, Brasília, DF, v. 18, n. 1, p. 9-16, 2007.

EIRAS, Suélen et al. Fatores de adesão e manutenção da prática de atividade física por parte de idosos. Revista Brasileira de Ciências do Esporte, Campinas, v. 31, n. 2 , p. 75-89, 2010.

FLETCHER, Gerald et al. Exercise standarts for testing and training: a statement for healthcare professionals from the American Heart Association. Circulation, Waltham, v. 104, n. 14, p. 1694-1740, 2001

FLORINDO, Alex et al. Fatores associados à prática de exercícios físicos em homens voluntários adultos e idosos residentes na grande São Paulo, Brasil. Revista Brasileira de Epidemiologia, São Paulo, v. 4, n. 2, p. 105-113, 2001.

GREENE, Laurence et al. Identifying dimensions of physical (motor) functional capacity in healthy older adults. Journal of Aging and Health, Thousand Oaks, v. 5, n. 2, p. $163-178,1993$.

HALPERN, Alfredo. A epidemia de obesidade. Arquivos Brasileiros de Endocrinologia e Metabologia, São Paulo, v. 43, n. 3, p. 175-176, 1999.

HOMBERGH, C. E. et al. Physical activities of noninstitutionalized dutch elderly and characteristics of inactive elderly. Medicine and Science in Sports and Exercise, Indianapolis, v. 27, n. 3, p. 334-339, 1995.

IYENGAR, Bellur. Yoga: the path to holistic health. London: Dorling Kindersley, 2001

INSTITUTO BRASILEIRO DE GEOGRAFIA E ESTATÍSTICA (IBGE). Síntese de indicadores sociais: uma análise das condições de vida da população brasileira. Rio de Janeiro: IBGE, 2008.

INSTITUTO BRASILEIRO DE GEOGRAFIA E ESTATÍSTICA (IBGE). Síntese de indicadores sociais: uma análise das condições de vida da população brasileira. Rio de Janeiro: IBGE, 2010

ISER, Betine et al. Fatores de risco e proteção para doenças crônicas não transmissíveis obtidos por inquérito telefônico - Vigitel Brasil - 2009. Revista Brasileira de Epidemiologia, São Paulo, v. 14, n. 1 (Supl.), p. 90-102, 2011.

KRUG, Rodrigo et al. Condições de saúde de idosos longevos participantes de um programa de atividades físicas. Revista Mineira de Educação Física, Belo Horizonte, Edição Especial, v. 3, n. 7, p. 1012-1021, 2012.

KUHNEN, Ana. Programa de exercícios físicos nos centros de saúde: as condições de saúde dos idosos. 2008. 103 f. Dissertação (Mestrado em Educação Física) Universidade Federal de Santa Catarina, Florianópolis, 2008.

MAZO, Giovana et al. Valores normativos e aptidão funcional em homens de 60 a 69 anos de idade. Revista Brasileira de Cineantropometria e Desempenho Humano, Florianópolis, v. 12, n. 5, p. 316-323, 2010. 
MERATI, Matteo et al. Physical activity effects in elderly female: evaluation through six-minute walking test. Sport Science Review, v. 20, n. 5-6, p. 25-56, 2011.

MINAYO, Maria. O envelhecimento da população brasileira e os desafios para o setor saúde. Cadernos de Saúde Pública, Rio de Janeiro, v. 28, n. 2, p. 208-209, fev. 2012

MINISTÉRIO DA SAÚDE. Vigitel Brasil 2008: vigilância de fatores de risco e proteção para doenças crônicas por inquérito telefônico. Brasília, DF: MS, 2009.

MOTA, J. et al. Atividade física e qualidade de vida associada à saúde em idosos participantes e não participantes em programas regulares de atividade física. Revista Brasileira de Educação Física e Esporte, São Paulo, v. 20, n. 3, p. 219-225, 2006.

NIEMAN, David. Exercício e saúde: como se previnir de doenças usando o exercício como seu medicamento. 1. ed. São Paulo: Manole, 1999.

NUNES, Maria et al. Influência das características sociodemográficas e epidemiológicas na capacidade funcional de idosos residentes em Ubá, Minas Gerais. Revista Brasileira de Fisioterapia, São Carlos, v. 13, n. 5, p. 376-382, 2009.

NUNES, Marcelo; SANTOS, Suely. Avaliação funcional de idosos em três programas de atividade física: caminhada, hidroginástica e Lian Gong. Revista Portuguesa de Ciências do Desporto, Porto, v. 9, n. 2-3, p. 150-159, 2009.

PAGOTTO, Valéria. Auto-avaliação do estado de saúde em idosos usuários do Sistema Único de Saúde de Goiânia-Goiás. 143 f. 2009. Dissertação (Mestrado em Enfermagem) - Universidade de Federal de Goiás, Goiânia, 2009.

PEREIRA, João; OKUMA, Silene. O perfil dos ingressantes de um programa de educação física para idosos e os motivos de adesão inicial. Revista Brasileira de Educação Física e Esporte, São Paulo, v. 23, n. 4, p. 319-334, out./dez. 2009

RIKLI, Roberta; JONES, Jessie. Functional Fitness Normative Scores for Community-Residing Older Adults, Ages 60-94. Journal of Aging and Physical Activity, Birmingham, v. 7, n. 2, p. 162-181, 1999.

RIKLI, Roberta; JONES, Jessie. Developmet and validation of criterion-referenced clinically relevant fitness standards for maintaining physical independence in later years. The Gerontologist, Oxford, v. 53, n. 2, p.1-13, 2012.

SILVEIRA, Erika et al. Prevalência e fatores associados à obesidade em idosos residentes em Pelotas, Rio Grande do Sul, Brasil: classificação da obesidade segundo dois pontos de corte do índice de massa corporal. Cadernos de Saúde Pública, Rio de Janeiro, v. 25, n. 7, p. 1569-1577, 2007.

SOARES, J.; SESSA, M. Medidas de força muscular. In: MATSUDO, Victor (ed.). Testes em ciência do esporte. 5. ed. São Caetano do Sul: Celafiscs, 1995. p. 52-72.

SOBREIRA, Francisca; SARMENTO, Weslley; OLIVEIRA, Ana. Perfil epidemiológico e sociodemográfico de idosos frequentadores de grupo de convivência e satisfação quanto à participação no mesmo. Revista Brasileira de Ciências de Saúde, João Pessoa, v. 15, n. 4, p. 429-438, 2011.

STREIT, Inês et al. Aptidão física e ocorrência de quedas em idosos praticantes de exercícios físicos. Revista Brasileira de Atividade Física e Saúde, Florianópolis, v. 16, n. 4, p. 347-52, 2011

TORRES, Michelle et al. Análise da qualidade de vida em idosos praticantes de atividade física regular em Terezina-Piauí. ConScientiae Saúde, São Paulo, v. 9, n. 4, p. 667-675, 2010 .

VERAS, Renato. A longevidade da população: desafios e conquistas. Revista Velhice e Envelhecimento, [s. I.], v. 24, n. 75, 2003. 
ata de Submissão: 30/10/2018

Data de Aprovação: 30/11/2018
VERAS, Renato. Experiências e tendências internacionais de modelos de cuidado para com o idoso. Ciência e Saúde Coletiva, Rio de Janeiro, v. 17, n. 1, p. 213-238, 2012.

VIRTUOSO, Janeisa et al. Perfil de morbidade referida e padrão de acesso a serviços de saúde por idosos praticantes de atividade física. Ciência e Saúde Coletica, Rio de Janeiro, v. 17, n. 1, p. 23-31, 2012.

WORLD HEALTH ORGANIZATION (WHO). Envelhecimento ativo: uma política de saúde. Brasília, DF: Organização Pan-Americana da Saúde, 2005.

ZAGO, Anderson. Exercício físico e o processo saúde doença no envelhecimento. Revista Brasileira de Geriatria e Gerontologia, Rio de Janeiro, v. 13, n. 1, p. 153-158, 2010. 